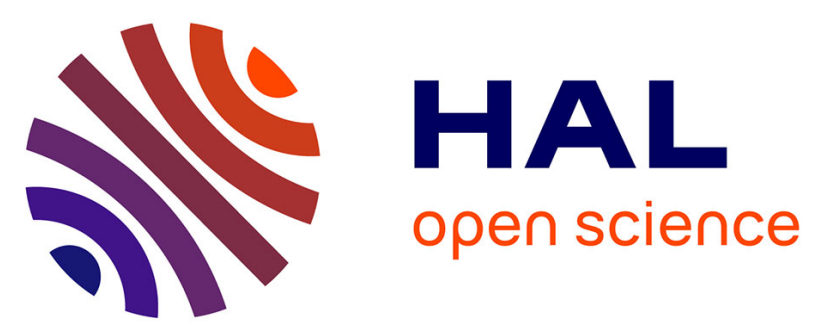

\title{
Building SSPs for climate policy analysis: a scenario elicitation methodology to map the space of possible future challenges to mitigation and adaptation
}

Julie Rozenberg, Céline Guivarch, Robert Lempert, Stéphane Hallegatte

\section{- To cite this version:}

Julie Rozenberg, Céline Guivarch, Robert Lempert, Stéphane Hallegatte. Building SSPs for climate policy analysis: a scenario elicitation methodology to map the space of possible future challenges to mitigation and adaptation. Climatic Change, 2014, 122, pp.509 - 522. 10.1007/s10584-013-0904-3 . halshs-01053726

\author{
HAL Id: halshs-01053726 \\ https://shs.hal.science/halshs-01053726
}

Submitted on 1 Aug 2014

HAL is a multi-disciplinary open access archive for the deposit and dissemination of scientific research documents, whether they are published or not. The documents may come from teaching and research institutions in France or abroad, or from public or private research centers.
L'archive ouverte pluridisciplinaire HAL, est destinée au dépôt et à la diffusion de documents scientifiques de niveau recherche, publiés ou non, émanant des établissements d'enseignement et de recherche français ou étrangers, des laboratoires publics ou privés. 


\title{
Building SSPs for climate policy analysis: a scenario elicitation methodology to map the space of possible future challenges to mitigation and adaptation
}

\author{
Julie Rozenberg ${ }^{1}$, Céline Guivarch ${ }^{1,2}$, Robert Lempert ${ }^{3}$, Stéphane Hallegatte ${ }^{4}$ \\ 1. CIRED, Nogent-sur-Marne, France \\ 2. Ecole des Ponts ParisTech, Champs-sur-Marne, France \\ 3. RAND, 1776 Main St., Santa Monica, CA, 90407, USA \\ 4. World Bank, Washington DC, USA
}

Pre-print from: Rozenberg, J., Guivarch, C., Lempert, R., Hallegatte, S. 2013. "Building SSPs for climate policy analysis: a scenario elicitation methodology to map the space of possible future challenges to mitigation and adaptation". Climatic Change (forthcoming). Special Issue on "A Framework for the Development of New Socio-economic Scenarios for Climate Change Research" edited by Nebojsa Nakicenovic, Robert Lempert, and Anthony Janetos. 10.1007/s10584-013-0904-3

\begin{abstract}
The scientific community is now developing a new set of scenarios, referred to as Shared Socioeconomic Pathways (SSPs) that will be contrasted along two axes: challenges to mitigation, and challenges to adaptation. This paper proposes a methodology to develop SSPs with a "backwards" approach based on (i) an a priori identification of potential drivers of mitigation and adaptation challenges; (ii) a modelling exercise to transform these drivers into a large set of scenarios; (iii) an $a$ posteriori selection of a few SSPs among these scenarios using statistical cluster-finding algorithms. This backwards approach could help inform the development of SSPs to ensure the storylines focus on the driving forces most relevant to distinguishing between the SSPs. In this illustrative analysis, we find that energy sobriety, equity and convergence prove most important towards explaining future difference in challenges to adaptation and mitigation. The results also demonstrate the difficulty in finding explanatory drivers for a middle scenario (SSP2). We argue that methodologies such as that used here are useful for broad questions such as the definition of SSPs, and could also be applied to any specific decisions faced by decision-makers in the field of climate change.
\end{abstract}

\section{A new generation of scenarios}

Most published analyses of climate change mitigation policy, and many investigating climate change impacts and adaptations, are based on long-term socioeconomic scenarios. Up to now, most analyses have used the Special Report on Emissions Scenarios (SRES, see IPCC, 2000), that describe possible world evolutions in demographic, social, economic, and technological terms, up to 2100.They assume that there are no climate change impacts and no mitigation policies, and thus have been used as "baselines" to assess the performance of adaptation and mitigation policies, and to investigate residual impacts from climate change. For instance, the costs and benefits of climate mitigation policies can be estimated by comparing the change in global mean temperature in a SRES scenario and in the same scenario in which specific climate policies are implemented (see a review in IPCC, 2007). Also, the potential impacts of climate change can be assessed by comparing a SRES scenario with the same scenario in which climate impacts are included (e.g., Arnell, 2004). 
The scientific community is now developing a new set of scenarios (Shared Socioeconomic Pathways or SSPs) to replace the SRES (Moss et al., 2010; van Vuuren et al., 2010; Kriegler et al., 2010; Arnell et al.,2011; O'Neill et al., 2012). As with the earlier SRES scenarios, the new SSPs will describe different socioeconomic characteristics, different vulnerabilities, and different GHG emissions. To assist with the exploration of both adaptation and mitigation questions with the same scenarios, Arnell et al. (2011) propose to develop SSPs that are contrasted along two axes: socio-economic challenges to adaptation and socio-economic challenges to mitigation.

Using "story and simulation" methods [Garb et al., 2008], similar to those used for SRES, groups of experts would build the SSPs working collaboratively to choose a common set of qualitative storylines, informed by experts' intuition regarding the most important driving forces leading to different vulnerabilities to climate change and different abilities to mitigate (see O'Neill, 2012 for an illustration with the SSP process). Analysts would then use computer simulation models to develop quantitative projections based on these storylines.

But this process may fail to yield storylines that focus on the most important driving forces for each SSP, and avoid focusing on the less important drivers. For some driving forces, the direction of the influence can be ambiguous a priori. For instance, it is likely that climate change vulnerabilities in an urban world are different from those in a rural world; but it is difficult to guess whether one is more or less vulnerable than the other. Cities are vulnerable to floods, air pollution and heat waves while rural zones are vulnerable to drought (falling yields), famines and migration. There is an obvious difference in the nature of vulnerability but not in the magnitude of the vulnerability. Moreover, some mechanisms (feedbacks, rebound effects, etc.) might appear negligible a priori but reveal themselves to be crucial in a more careful analysis. This is why an a priori selection of the likely drivers of the capacity to mitigate and adapt appears insufficient to us, and could be completed by an analysis that validates the most important drivers a posteriori.

Furthermore, since future socioeconomic and environmental changes are highly uncertain, policies need to be tested against a variety of scenarios that cover a large range of possible futures (Lempert 2012; Hallegatte, 2009). It would indeed be dangerous to implement a climate policy that performs well in one given scenario but completely fails in another, for instance if the population is larger or technological change slower than expected. Although the "story and simulation" process creates coherent scenarios through the storylines, it does not guarantee that the scenario outcome in terms of GDP or GHG emissions for instance will cover the uncertainty spectrum expected by users.

To address these challenges, we suggest here developing SSPs using a "backwards" approach. Our methodology is based on (i) an identification of potential drivers of challenges to mitigation and adaptation, (ii) a modeling exercise to explore the uncertainty space and select scenarios, and (iii) an $a$ posteriori confirmation of which drivers matter and of the sign of their influence on adaptation and mitigation challenges, using statistical cluster-finding algorithms.

In recent years, several research groups have employed similar approaches, in which cluster-finding algorithms applied to databases of multiple simulation model runs are used to suggest scenarios relevant for specific policy questions with less potential ambiguity than can arise from story and simulation methods [Gerst et al., 2013; Groves and Lempert, 2007; Haasnoot et al., 2013; Hamarat et al., 2013; Kasprzyk et al., 2013; Robert J. Lempert et al., 2006; McJeon et al., 2011]. For example, one such "scenario discovery" analysis focused on mitigation strategies, evaluating the costs and benefits of a Renewable Energy Portfolio standard in the U.S. It found that the availability of low-cost biomass feedstock and low-cost sites for wind energy were the most important drivers for whether or not the policy produced high cost outcomes (Toman et al., 2008). The concept of "backwards" analysis 
is similar to the "tolerable window" approach (Petschel-Held et al, 1999) that identified policies consistent with defined guardrails for climate evolution, though the current approach can be used with any forward-running model [Lempert et. al. 2006]. The concept of defining scenarios relevant to specific decision-relevant criteria is also consistent with the Global Business Network's "strategic scenarios" approach, although we do not start with a specific decision but with the broader issue of defining scenarios that are contrasted in terms of future challenges to adaptation and mitigation, similarly to recent work on defining scenarios for climate adaptation studies [Birkmann et al., 2013]. While the structural form of our model limits the results described in this paper, our approach could usefully be generalized to employ multi-model analyses (Barker et al., 2002; Barker et al., 2006; Peace and Weyant, 2008).

To build a database of many cases in this study, we first identify potential drivers of future challenges to adaptation and mitigation and translate some of our potential drivers into different model parameters (e.g., the amount of ultimately recoverable oil resources). Other drivers cannot be accommodated in the model and are considered part of a narrative, accompanying model parameters and results (e.g. quality of governance). Combining the different states of these drivers (e.g., the amount of oil is low or large), we build a database of model runs (as in Rozenberg et al., 2010). We then obtain several hundreds of scenarios ${ }^{1}$ by combining the model outputs with additional external drivers that cannot be included in the model, but that are essential for an analysis on future challenges to adaptation and mitigation.

Once we have constructed the database, we distinguish the scenarios using criteria measuring future challenges to adaptation (e.g., the share of people living below the poverty line) and mitigation (e.g., baseline $\mathrm{CO}_{2}$ emissions). The scenario discovery algorithms then identify the common characteristics (e.g., the demographic changes, the extent of globalization) that best predict the scenarios where the challenges to adaptation or mitigation are high or low. They help us select a few SSPs that are contrasted along these criteria.

For SSPs, the selected scenarios have to be sufficiently general, since they need to be applicable to a large set of research questions and decisions. Therefore, instead of starting from a specific decision, we look to find contrasted scenarios in terms of adaptation and mitigation challenges. But this "scenario elicitation" methodology can be used to build different sets of scenarios, each decision maker choosing the drivers relevant to a particular policy issue and selecting his or her own scenarios from a common database.

To summarize, we propose the following approach for developing SSPs:

(i) We first identify a priori the main driving forces of the world future challenges to mitigation and adaptation to climate change, based on existing literature (Section 2).

(ii) We then translate these driving forces into model parameters for a global energy-economic model, and we combine these parameters to build a large number of model runs. We also

\footnotetext{
${ }^{1}$ Note that there is no consensus of the terminology used in scenario analysis. Here, we label each of our model runs a "scenario." The Robust Decision-Making tradition (e.g., Lempert and Groves, 2010) labels these runs "cases" and considers a "scenario" as a set of "case" particularly relevant to the analysis of a given decision.
} 
combine model outputs with external drivers (that are not included in the model) to create a large set of scenarios (Section 3$)^{2}$

(iii) We analyze the resulting database using indicators measuring future challenges to mitigation and adaptation, and we identify a posteriori the main driving forces of these future challenges. Then we select five contrasting combinations of drivers to cover the range of possible challenges to adaptation and mitigation (Section 4).

The last section discusses how this could define a new approach to the use of scenarios by the climate community. Noting that the most relevant scenarios are often crafted explicitly for specific communities facing specific climate-related decisions (European Environment Agency, 2009; Parson et al., 2006), this new concept envisions providing data and tools that would allow each user to construct his or her own scenarios as needed, while ensuring broad consistency among users with the underlying base of scientific knowledge.

\section{The a priori drivers of challenges to adaptation and mitigation}

Hallegatte et al. (2011) propose three dimensions to explore climate change vulnerability and adaptation challenges, that are described in details in Section I of the supplementary material: (i) Globalization: a "converging" world vs. a "fragmented" world; (ii) Equity: inclusive development vs. "growth and poverty" development; and (iii) Environmental stress: an "environment-oriented" world vs. an "environmentally-stressed" world. As described in the Supplementary material, these dimensions, along with dependency on fossil fuel, also appear highly relevant to challenges to mitigation.

The resulting four dimensions are shown in Figure 1, giving an idea of which parameters can be included in each dimension. The figure suggests that some of these parameters can be included in different dimensions (e.g., urbanization can be included in the environmental/lifestyle dimension or in the convergence dimension), showing that there will always be some flexibility and subjectivity in how our approach is applied. Note also that the literature is not the only place to look for a priori drivers of future adaptation and mitigation challenges. Other sources such as expert elicitation surveys (Schweizer and O'Neill, this issue) could also prove valuable. Nonetheless, the drivers considered here should prove more than sufficient for the illustrative purposes of this paper.

\footnotetext{
${ }^{2}$ The scenario discovery literature generally refers to the entries in the database of model results as cases. Here we use the term scenarios because we have added to the database entries information associated with narratives in addition to the results of model runs.
} 


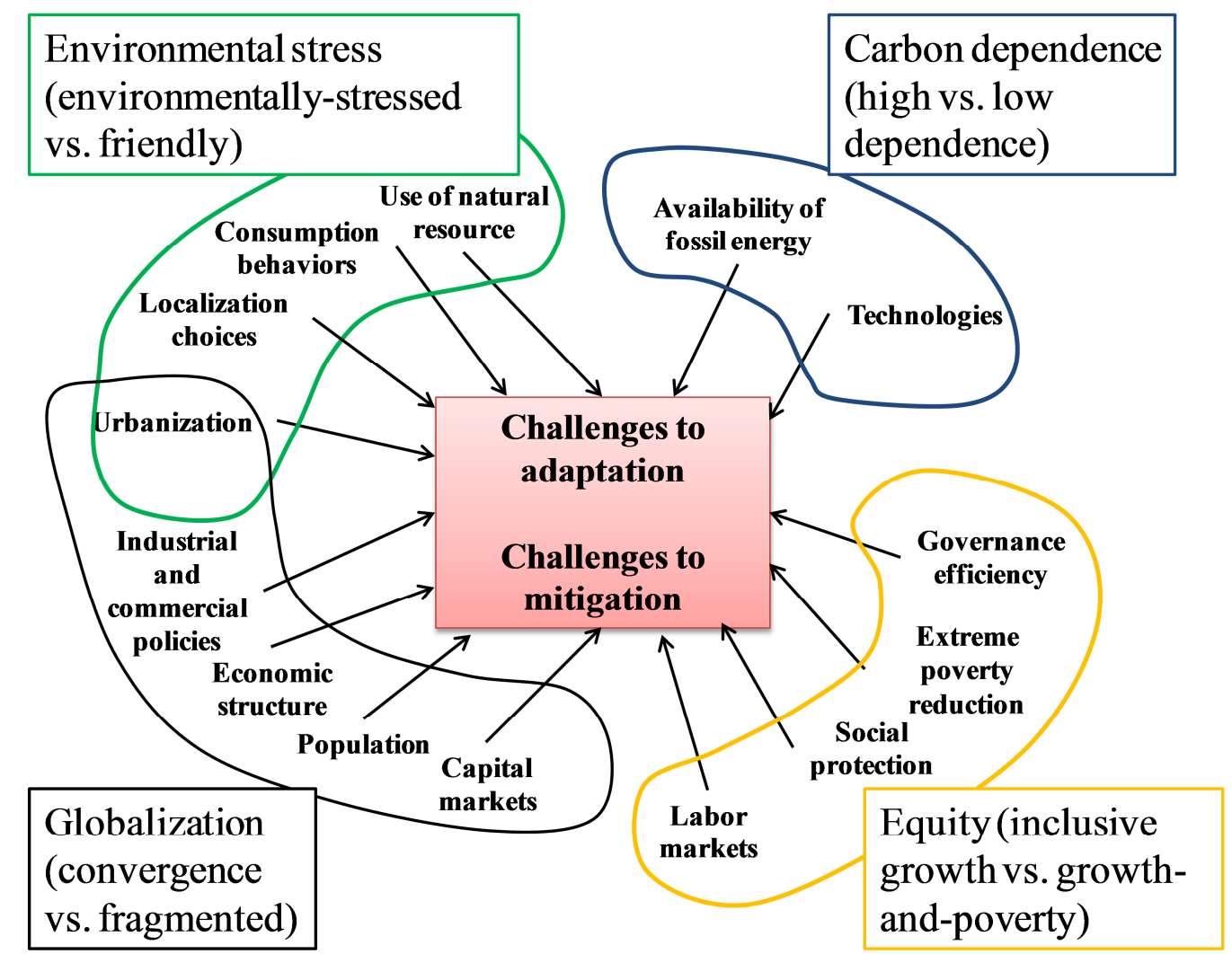

Figure 1: Identified drivers of the challenges to mitigation and adaptation, in the four main dimensions.

\section{How to build scenarios}

There are good reasons to think that these factors represent the major drivers of challenges to adaptation and to mitigation, but this is only an informed guess. Complex mechanisms, interactions, and feedbacks can act on these drivers, and a more sophisticated analysis is possible. To test whether these drivers are well chosen, we translated some of them into model parameters.

To do so, we used the IMACLIM-R model (Rozenberg et al, 2010; Waisman et al, 2012 and Section II in the supplementary material), which projects the long-term evolution of the world economy and allows us to explore the uncertainty that arises from unknown exogenous trends (e.g., future population) and parameter values that are debated.

For a given model, not all drivers can be translated into input parameters. Indeed, some of the drivers are direct inputs of the IMACLIM-R model (e.g., population), some are the result of upstream hypotheses (e.g., the availability of fossil energy is the result of hypotheses on oil resources and inertia in the development of production capacities), and some are model outputs (e.g., economic structure). Note that some potential technological breakthrough such as hyper efficient micro grids or black swans like epidemics are not modelled here.

For this exercise, we selected the following drivers to be translated into input parameters of the IMACLIM-R model and consider several alternative values for these parameters to reflect uncertainty on future challenges to adaptation and mitigation: 


\section{Dimension1: Globalization}

Population. We use the three UN scenarios (low, median, and high). In a next step, population data defined specifically for the SSPs could be used.

Economic structure. Even though this driver is an output of the IMACLIM-R model, we influence it by introducing three assumptions on the speed of labor productivity convergence (see III.1 in the supplementary material). This parameter influences economic growth in each region, but note that GDP growth is endogenous in IMACLIM-R.

Capital markets. The IMACLIM-R model treats capital balances as exogenous, so we consider two assumptions about global financial imbalances reduction: In the first assumption financial imbalances are phased out exponentially in two decades, whereas in the second assumption they remain constant for the whole simulation period.

\section{Dimension2: Environmental stress}

Energy sobriety. We make two assumptions (i.e. two groups of hypotheses affecting many different variables) regarding energy sobriety:

- Development patterns: We introduce two assumptions on the evolution of households' preferences (environmentally-inclined or energy-intensive) in transportation and housing (evolution of the number of cars per capita, maximum dwelling surface per capita in developing countries) as well as on the saturation level of households' industrial goods consumption (see III.2 in the supplementary material).

- Production choices: We introduce two alternatives on the freight content of economic growth through alternative evolutions of the input-output coefficient representing the transportation requirement per unit of good produced (see III.2 in the supplementary material).

- Induced energy efficiency: Even though energy efficiency is driven by energy prices, we introduce two alternatives for the parameters describing its maximum annual improvement in the leading country and the catch-up speed of the others (see III.2 in the supplementary material).

\section{Dimension3: Carbon supply}

Availability of fossil energy. We introduce two assumptions about oil resources (parameters include the amount of ultimately recoverable resources, inertia in the deployment of non conventional oil, the maximum growth rate of Middle-East production capacities), the gas price indexation on the oil price, and the elasticities of coal price growth to demand changes (see III.3 in the supplementary material). Each of these variables can take two different values depending on the assumption.

Availability of low-carbon technologies. We build two assumptions for parameters describing the market penetration of nuclear energy, renewable resources, carbon capture and storage, and electric vehicles. These parameters include learning rates and maximum market shares throughout the simulation period. (More details are given for each technology in III.3 in the supplementary material.)

\section{Dimension4: Equity}


Dimension 4 has to be treated differently, because IMACLIM-R in its current form cannot include its drivers (inequality within countries). Since the model is based on a representative consumer-worker, it cannot take into account income distribution among consumers-workers. Considering the importance of this driver, it cannot be disregarded. We thus introduce it through an external driver, i.e. that is not included in the model and does not modify the model outputs, but adds numerical information to the model results to build a scenario.

In the current case, therefore, we add to the model outputs quantitative information on "equity." Some of the scenarios are built assuming a global reduction of within-country inequality (an "inclusive growth" set of scenarios), in which the share of income of the $20 \%$ poorest in countries increases by $33 \%$ by 2090 (e.g., in a country where the $20 \%$ poorest receive an income corresponding to $6 \%$ of total GDP in 2010, this share increases to $8 \%$ in 2090). Others are built assuming a global increase in within-country inequality, with a share of income of the $20 \%$ poorest that decreases by $33 \%$ by 2090 . To the model outputs, therefore, we add an additional variable, namely the income of the $20 \%$ poorest, which is built from model outputs (GDP per capita in less developed countries) and from this external driver.

The result is a set of 286 scenarios $^{3}$ (see Figure 2 below and Figure 1 in the supplementary material), each being the combination of (1) a set of model parameters describing the drivers, (2) a model run with these parameters, and (3) additional quantitative and qualitative information that cannot be accommodated in the model but are relevant for challenges to adaptation and mitigation (e.g., in our case, inequalities within countries).

GDP per capita of the $20 \%$ poorest in developing countries in 286 scenarios

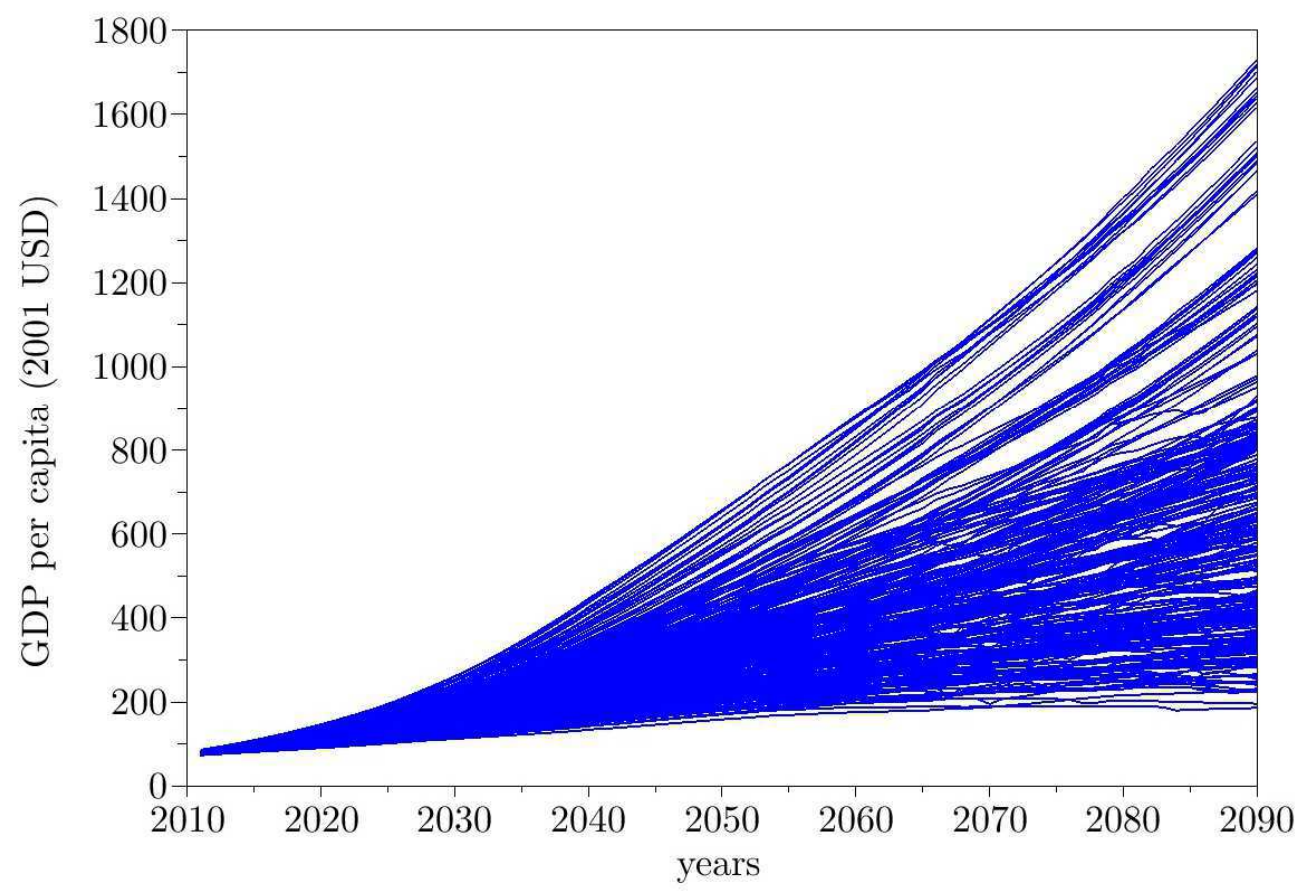

\footnotetext{
${ }^{3}$ Combining all assumptions creates 288 model runs, but one baseline did not run until the end of the simulation period. Thus, two scenarios are excluded from the database (derived from this model run and the two hypotheses on equity).
} 
Figure 2: GDP per capita of the $20 \%$ poorest in a selection of developing countries in 286 scenarios resulting from 143 model runs with IMACLIM-R and two hypotheses on the share of income of the $20 \%$ poorest

\section{How to select relevant scenarios}

To select scenarios that cover challenges to mitigation and adaptation, the first step is to define indicators for these challenges. This is a very important and difficult endeavor. Much work has been devoted to this task, but there is little agreement on how to proceed. Taking the example of the challenges to adaptation, Füssel (2009) reviews the many indicators that have been proposed and shows that they lead to very different prescriptions and vulnerability hot spots. Also, he shows that vulnerability and adaptive capacity cannot be identified in isolation from political considerations and value and ethical judgments. Our analysis is thus developed and illustrated using very simple indicators (two for mitigation and two for adaptation), taking into account the fact that more work on this issue needs to be done, and that the methodology needs to be able to accommodate a fairly large set of indicators.

For mitigation, we chose baseline $\mathrm{CO}_{2}$ emissions as an indicator. We are well aware that this measure does not include all components of the challenges to mitigation. For instance, good governance and reduced inequalities are likely to make it easier to implement mitigation policies, regardless of $\mathrm{CO}_{2}$ emissions. But as a first-step analysis, we use this indicator. In a second step, we use the GDP losses from a mitigation policy implemented to stabilize radiative forcing at a given level.

For adaptation, no natural indicator is available. Still well aware of the limits, we decided to use the income of the $20 \%$ poorest in a selection of developing countries (African countries, India, South America [except Brazil] and South East Asia). Of course, this is a very partial indicator, and it is well known that challenges to adaptation will depend on many other factors, such as governance and technologies (see the review in the supplementary material and in Hallegatte et al., 2011). In the current analysis, we use this simple indicator only to illustrate our methodology and make a first proposal for SSPs. In a second step, we use the share of jobs in agriculture in developing countries. 


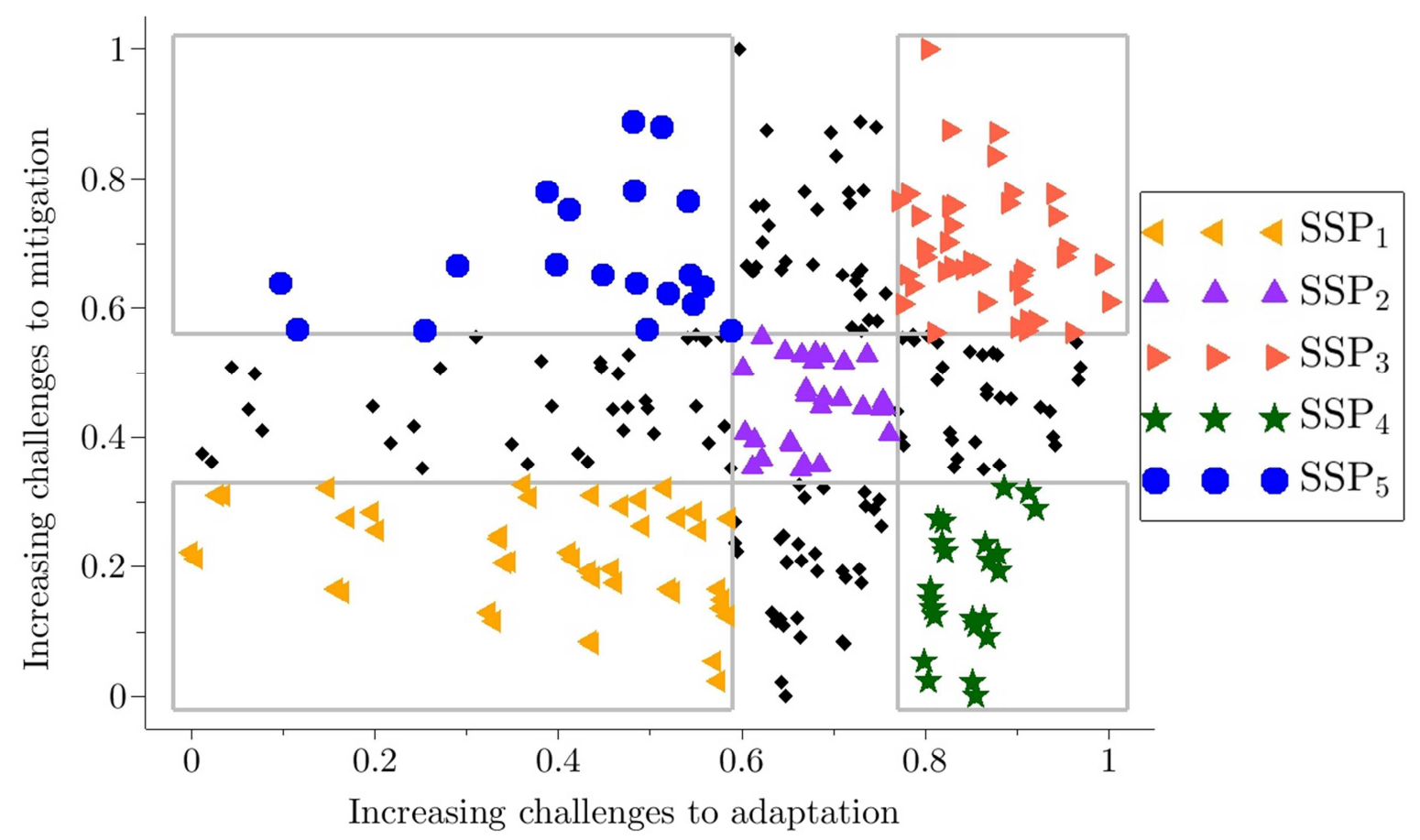

Figure 3: Capacities to adapt and to mitigate that define the five SSP spaces and the results of our 286 model runs arrayed in this space.

We next normalize our two indicators (the sum of global emissions over the 2011-2090 period for challenges to mitigation and the discounted income of the $20 \%$ poorest in developing countries, over the same period, for challenges to adaptation) and map our 286 scenarios over the resulting space. As shown in Figure 3, the scenarios span most combinations of challenges to mitigation and adaptation as defined by these indicators. We then define five regions in this space that correspond to the five SSPs.

In the selection of SSP spaces, we emphasize contrast, i.e., on having scenarios with different challenges to mitigation and adaptation. We do not focus on the "probability" of these scenarios. The "plausibility" is supposed to be ensured in the first phase of this analysis, when the determinants have been chosen and transformed into model parameters. We do not want to focus on probabilities because the ability to assess them appears out of reach and because focusing on the most likely scenarios would lead to disregarding low-probability high-impact scenarios, which might be the most relevant in a risk-management approach. Since we think that the analysis of climate policies is an analysis of climate risks more than anything else, the inclusion of low-probability scenarios in SSPs appears essential.

To select the five SSP boxes, we choose numerical thresholds for the challenges to mitigation and adaptation indicators that characterize each SSP. Here we choose the two thresholds so that one-third of the scenarios lie below the first and one-third lie above the second (see Figure 3). This choice results in a significant number of scenarios in each SSP region. One could, however, set thresholds independently of the scenarios distribution, for instance by requiring that all SSP regions have the same area. As described below, we find that in this particular example our results are relatively insensitive to the choice of thresholds. More generally, the choice of threshold might usefully be assisted by approaches such as the hierarchical cluster methods used by Gerst et. al. [2013]. 
Having chosen thresholds to define the SSP regions, we can then use a "scenario discovery" cluster analysis to identify the main drivers of each scenario group. "Scenario discovery," often used to support robust decision making (Robert Lempert and Kalra, 2011; R J Lempert et al., 2003), provides a computer-assisted method of scenario development that applies statistical or data-mining algorithms to databases of simulation model results to characterize the combinations of uncertain inputs parameter values most predictive of specified classes of results. Importantly, scenario discovery also suggests which uncertain input parameters have less influence. We apply a modified version of the PRIM (Patient Rule Induction Method) (Friedman and Fisher, 1999) to the 286 scenarios spanning the range of adaptation and mitigation indicators shown in Figure 3. A variety of classification algorithms have been used for scenario discovery. PRIM often proves useful in this context because it facilitates users' ability to trade between the accuracy of the scenario descriptions and their interpretability, that is, the ease with which decision makers can understand the information contained in the scenarios (see for example, the comparison between PRIM and the Classification and Regression Tree (CART) algorithm in Bryant, Lempert, and Bankes [2008]).

An SSP is defined by a range of values for the adaptation and mitigation indicators. PRIM seeks a set of drivers that maximize the matching between those drivers and the model scenarios that have the indicator values associated with each SSP. For instance, for SSP5, we want to find the drivers such that a scenario with these drivers has a high likelihood of being in the upper-left-hand corner of Fig 5 and such that a scenario is in the upper-left-hand corner has a high likelihood of having these drivers. To measure this match, we use three criteria (see Bryant and Lempert, 2010). Coverage is the fraction of scenarios consistent with the indicators (e.g. that lie in the SSP5 corner of Fig 5) that are also consistent with the drivers. Density is the fraction of all scenarios consistent with the drivers that are also consistent with the indicators. ${ }^{4}$ Interpretability represents that ability of decision makers to understand the information by the combinations of drivers and is measured by having a small number of drivers.

Since these three measures are generally in tension with one another, PRIM provides the user a set of options representing different tradeoffs among density, coverage, and interpretability.

Table 1 shows our results. Each row shows an SSP and the middle eight columns list its potential drivers. A cell filled with black text indicates that a driver plays a significant role in that SSP whereas grey italics text indicates that the driver plays a partial role. We distinguish the former from the latter using the resampling test described in Bryant and Lempert (2010). This test runs PRIM on multiple subsamples of the original dataset and notes the fraction of subsamples for which each parameter emerges as an important driver of the scenario definition. We consider a driver that scores greater than $50 \%$ in the test as significant and less significant otherwise.

The final column shows the explanatory power of these combinations of drivers, as measured by their coverage and density (rounded at 5\%). For instance, the SSP4 row shows that low equity, slow convergence, and high energy sobriety contribute most significantly to SSP4 while medium or low population plays a partial role. The last column says that ninety percent of the cases in the region of Figure 3 noted as SSP4 meet these conditions (coverage) and ninety percent of the cases that meet these conditions are SSP4 (density).

\footnotetext{
${ }^{4}$ Coverage is analogous to "sensitivity" or "recall" in the classification and information retrieval literatures. Density is analogous to "precision" or "positive predictive value" in those literatures.
} 
Some drivers, such as equity, contribute strongly to all the SSPs. Indeed, this driver has a direct impact on the challenges to adaptation axis, since it was used to calculate the indicator (see Section 4); this driver splits the income of the $20 \%$ poorest into two groups, with a compression to the right as GDP per capita decreases. In the same way, the "energy sobriety" driver has a strong impact on challenges to mitigation, since it directly influences $\mathrm{CO}_{2}$ emissions in the baseline. It also influences the challenges to adaptation because energy sobriety leads to higher GDP, i.e. to less poverty. ${ }^{5}$

The impact of population on the indicators is ambiguous and not always significant. Although one might think that a higher population would reduce GDP per capita in a world with finite resources, a higher population growth rate implies higher potential economic growth in the model, so that challenges to adaptation might decrease. Moreover, higher economic growth accelerates capital turnover and increases the share of low-carbon technologies, thus decreasing challenges to mitigation. The results show, however, that a high population is inconsistent with SSP1 and that a low population is inconsistent with SSP3.

Other drivers, such as fossil fuel availability and capital markets, contribute to few if any SSPs. The non-significant impact of fossil fuel availability is due to two contradictory effects: on the one hand, a constrained oil supply induces substitution toward coal, which emits more $\mathrm{CO}_{2}$ for the same energy service. On the other hand, it also induces higher energy prices, which trigger faster energy efficiency. In the same way, low-carbon technologies contribute to only one SSP because they tend to slow down energy efficiency through lower energy prices, which lessens their effect on carbon emissions.

An interesting result of this analysis is the difficulty in finding scenarios corresponding to SSP5 (i.e., scenarios with both high baseline emissions and high GDP growth). Indeed, to include one-third of all scenarios above the first threshold for adaptation challenges, we had to define it at a high value, around 0.6 (see Figure 3). As already mentioned, GDP growth is affected by energy consumption and sobriety. If energy consumption is too high - and energy efficiency too low - energy prices are so high than GDP growth is significantly reduced, especially in developing countries. This explains why there are few scenarios with high economic growth and high baseline emissions. This result however depends on the indicator considered, and taking a different indicator for challenges to mitigation helps finding SSP5 scenarios (see section III in the supplementary material).

Another conclusion of the analysis is the difficulty to define a unique SSP 2. Indeed, coverage only reaches $45 \%$, which means that $55 \%$ of the scenarios identified as potential SSP2 in Figure 3 corresponds to other drivers than those identified in Table 1. In other terms, there are other sets of drivers that could lead to scenarios with medium challenges to adaptation and mitigation. This quantitative result is consistent with discussions in the Boulder meeting in November 2011 (O'Neill et al., 2012) and the proposition to have several versions of SSP2.

\footnotetext{
${ }^{5}$ The "energy sobriety" driver contains hypotheses on behaviors, localization choices, and the potential for energy efficiency (energy efficiency is endogenous and driven by energy prices). In scenarios with high energy sobriety, energy prices are lower, accelerating GDP growth. This result warns against the use of exogenous GDP scenarios, developed independently from natural resources and energy modeling.
} 


\begin{tabular}{|c|c|c|c|c|c|c|c|c|}
\hline & $\begin{array}{c}\text { Equity } \\
\text { (2 options) }\end{array}$ & $\begin{array}{c}\text { Conver- } \\
\text { gence } \\
\text { (3 options) }\end{array}$ & $\begin{array}{c}\text { Energy } \\
\text { sobriety } \\
\text { (2 options) }\end{array}$ & $\begin{array}{l}\text { Availability } \\
\text { of low C } \\
\text { technologies } \\
\text { (2 options) }\end{array}$ & $\begin{array}{l}\text { Availability } \\
\text { of fossil } \\
\text { fuels } \\
\text { (2 options) }\end{array}$ & $\begin{array}{l}\text { Population } \\
\text { (3 options) }\end{array}$ & $\begin{array}{c}\text { Capital } \\
\text { markets } \\
\text { (2 options) }\end{array}$ & $\begin{array}{l}\text { Coverage/ } \\
\text { Density }\end{array}$ \\
\hline $\begin{array}{c}\text { SSP1 } \\
\text { (15\% of } \\
\text { cases) }\end{array}$ & improved & $\begin{array}{l}\text { Medium } \\
\text { or fast }\end{array}$ & high & & & $\begin{array}{l}\text { Medium } \\
\text { or low }\end{array}$ & & $\begin{array}{l}70 \% / \\
65 \%\end{array}$ \\
\hline $\begin{array}{l}\text { SSP2 } \\
(10 \% \text { of } \\
\text { cases) }\end{array}$ & improved & $\begin{array}{l}\text { Medium } \\
\text { or slow }\end{array}$ & low & & & low & & $\begin{array}{l}45 \% / \\
40 \%\end{array}$ \\
\hline $\begin{array}{l}\text { SSP3 } \\
(14 \% \text { of } \\
\text { cases) }\end{array}$ & worsen & & low & low & & $\begin{array}{l}\text { High or } \\
\text { medium }\end{array}$ & & $\begin{array}{l}80 \% / \\
65 \%\end{array}$ \\
\hline $\begin{array}{l}\text { SSP4 } \\
\text { (8\% of } \\
\text { cases) }\end{array}$ & worsen & slow & high & & & $\begin{array}{l}\text { Medium } \\
\text { or low }\end{array}$ & & $\begin{array}{l}90 \% / \\
90 \%\end{array}$ \\
\hline $\begin{array}{l}\text { SSP5 } \\
\text { (7\% of } \\
\text { cases) }\end{array}$ & improved & fast & low & & & & $\begin{array}{c}\text { Reduced } \\
\text { imbalances }\end{array}$ & $\begin{array}{l}50 \% / \\
75 \%\end{array}$ \\
\hline
\end{tabular}

Table 1: Combinations of future challenges to adaptation and mitigation in our five SSP spaces as identified by the scenario discovery analysis described in the text. Black/grey italics text indicates more/less important drivers. Coverage and density (rounded) measure the explanatory power of the drivers for each SSP.

Eventually, this representation allows testing the ex-post relevance of our drivers on our indicators. The hypotheses that we made about financial markets and the future reduction of imbalances are not $a$ posteriori main drivers of the world challenges to adaptation and mitigation, except for SSP5. These results must be moderated, however, because they depend significantly on the choice of indicators and on the delimitation of the SSP spaces.

As an illustration, we repeat the analysis using a different set of indicators. As an indicator of future challenges to mitigation, we calculate the GDP losses from a mitigation policy reducing emissions to stabilize radiative forcing at a given level (we retain a target at $3.7 \mathrm{Wm}^{-2}$ ). For adaptation challenges, we use the share of jobs in agriculture in developing countries.

The results of this second analysis share some similarities with those of the first one (see Table SP4 in the supplementary material); for example, convergence is slow for SSP4 and fast for SSP5; energy sobriety is high for SSP1 and low for SSPs 3 and 5. But significant differences can also be noted. For instance, low-carbon technologies become significant in SSPs 1, 3, 4 and 5. This is due to the mitigation challenges indicator (i.e., the cost of a mitigation policy): low-carbon technologies help meet the climate objective at a lower cost without slowing down energy efficiency, which is triggered by the carbon tax in all scenarios. In this second analysis, capital markets are significant in three SSPs because they have an impact on the adaptation challenges indicator: a reduction of financial imbalances (as in SSP1 for instance) induces developing countries to invest more locally. This accelerates structural change and reduces the share of jobs in agriculture.

The similarities and differences in these two analyses, with different criteria for challenges to mitigation and adaptation, constitute a first indication of the robust results for SSPs development. To 
go further, we consider only scenarios that are in the same SSP domain for both sets of indicators. For each SSP domain, only about ten scenarios are stable to a change of indicators, and they have a few drivers in common:

- SSP1 (11 scenarios): these scenarios are characterized by a high availability of low-carbon technologies, energy-sober behaviors and a reduction in financial imbalances.

- SSP2 (5 scenarios): there is no common characteristic to those scenarios.

- SSP3 (7 scenarios): these scenarios are the exact opposite of the SSP1 scenarios, and are characterized by a low availability of low-carbon technologies, energy-intensive behaviors and maintained financial imbalances.

- SSP4 (11 scenarios): these scenarios are characterized by low equity, low convergence and maintained financial imbalances.

- SSP5 (10 scenarios): these scenarios all have a high convergence, nine of them have a low availability of low carbon technologies and nine of them have reduced financial imbalances.

These results confirm that it is difficult to find a unique set of drivers defining SSP2; this intermediate scenario can be represented by very different futures. They also show that the relevant set of drivers depend on which SSP is considered. Interestingly, SSP1 and SSP3 are defined by the same set of drivers - but with opposite values - that mainly describes the energy system (technologies and energy intensity). Conversely, SSP4 and SSP5 are characterized by assumptions on socio-economic aspects, and particularly equity and economic convergence.

To test sensitivity to the choice of threshold for the SSP regions, we repeated the analysis a third time using same-sized SSP domains with a diamond-shaped domain corresponding to SSP2 ${ }^{6}$ (Fig. SP4 and Table SP5 in Supplementary Material). As the new domains include all scenarios, the number of explanatory drivers is lower than in the first analysis, and since the domains are moved to the left compared to the distribution, the equity driver is no longer significant for SSP3 and SSP4. The results however share similarities with those of the first one, in particular for SSP1 and SSP5 that have three constant drivers. The only contradictory result is for SSP2: improved equity was a significant driver in the first analysis while worsen equity is now significant. Note that here again, coverage is bad $(45 \%)$ for SSP2, which confirms that it is very difficult finding a storyline for this SSP.

This analysis remains preliminary and the robustness of its results would be increased by the inclusion of additional driver values, additional drivers, alternative selection criteria, and more models. In particular, combining different models with different designs would be key to improve our confidence in the SSP determinants, because it would help assess the uncertainties surrounding the model structures. Indeed, the coherence that we find for a certain set of drivers depends on modelling choices and might be called into question by other models.

\section{Conclusion and further challenges}

A central goal of scenario exercises is to inform the development of robust strategies, that is, strategies that perform better than the alternatives over a wide range of plausible future states of the world. When this process is successful, it can be surprising that uncertain factors do not affect the relative performance of a robust strategy.

\footnotetext{
${ }^{6}$ We credit the idea for a diamond-shaped domain corresponding to SSP2 to Jae Edmonds of the GCAM modelling group at the Joint Global Change Research Institute.
} 
Such context dependence of the most important scenarios for decision making presents both an opportunity and a challenge for the choice of socioeconomic scenarios. The opportunity is that it provides a clear definition of the most policy-relevant scenarios - those that most directly inform the tradeoffs among strategies. But it also suggests the need for different sets of scenarios for different decisions.

But as a first step, we propose in this paper an illustration of how this methodology could be applied to define a first set of SSPs, appropriate for a broad range of decisions concerning climate policies, including mitigation and adaptation. We show that this approach allows for the development of five baseline SSPs that have very different challenges to adaptation and mitigation (at least according to the very simple indicators we used in this study).

Most importantly, the development of SSPs will not be a one-shot exercise. Instead, it will be a continuous process of refinement. The methodology that is proposed here could be applied to an increasingly large set of scenarios produced using different methods and models. The methodology would indeed be particularly relevant if a large number of diversified models are used to construct the scenario database in which SSPs are selected. Also, it would be particularly useful to develop a large set of selection criteria, which could be used to select SSPs that are particularly adapted to a specific problem.

Over the long term, one can imagine a large multi-model scenario database and a diversity of selection criteria that can be used to identify which scenarios are most relevant for a given category of decisions. A web-based tool could then be proposed to allow decision makers to select the few scenarios that they need to consider in their decision making process. Such a tool would benefit from the multiplicity of models available in the literature. It would also avoid the difficult selection of a few marker scenarios, which will inevitably oversimplify the reality and result in the discarding of a lot of useful information.

\section{References}

Arnell N. W. (2004), Climate change and global water resources: SRES emissions and socio-economic scenarios, Global Environmental Change, 14(1), 31-52

Arnel N. W, T. Kram, T. Carter, K. Ebi, J. Edmonds, S. Hallegatte, E. Kriegler, R. Mathur, B. O'Neill, K. Riahi, H. Winkler, D. van Vuuren, T. Zwickel, A framework for a new generation of socioeconomic scenarios for climate change impact, adaptation, vulnerability, and mitigation research.

T. Barker, J. Koehler, and M. Villena. The costs of greenhouse gas abatement: A meta-analysis of post-SRES mitigation scenarios. Environmental Economics and Policy Studies, 5 :135,166, 2002

T. Barker, M. S Quereshi, and J. Koehler. The costs of greenhouse gas mitigation with induced technological change : A meta-analysis of estimates in the literature. 4CMR, Cambridge Centre for Climate Change Mitigation Research, 2006.

Bryant, B. P., and R. J. Lempert (2010), Thinking inside the box: A Participatory, computer-assisted approach to scenario discovery, Technological Forecasting and Social Change, 77, 34-49.

European Environment Agency (EEA) (2009), Looking back on looking forward: a review of evaluative scenario literature Rep. ISSN 1725-2237 European Environmental Agency, Copenhagen. 
Friedman, J. H., and N. I. Fisher (1999), Bump Hunting in High-Dimensional Data, Statistics and Computing, 9, 123-143.

Füssel H.-M. (2009), Review and quantitative analysis of indices of climate change exposure, adaptive capacity, sensitivity, and impacts. Background note to the World Development Report 2010, World Bank,Washington, D.C.

Garb, Y., S. Pulver and S. D. VanDeveer (2008). "Scenarios in society, society in scenarios: toward a social scientific analysis of storyline-driven environmental modeling." Environ. Res. Lett. 3: 1-8.

Gerst, M. D., P. Wang and M. E. Borsuk. (2013). "Discovering plausible energy and economic futures under global change using multidimensional scenario discovery. ." Environmental Modelling \& Software. 44: 76-86.

Haasnoot, M., J. H. Kwakkel, W. E. Walker and J. ter Maat (2013). "Dynamic adaptive policy pathways: A method for crafting robust decisions for a deeply uncertain world." Global Environmental Change 23(2): 485-498.

Hallegatte, S., 2009: Strategies to adapt to an uncertain climate change, Global Environmental Change $19,240-247$.

Hallegatte, S., V. Przyluski, A. Vogt-Schilb (2011), Building world narratives for climate change impact, adaptation and vulnerability analyses. Nature Climate Change, 1(3), p.151-155.

Hamarat, C., J. H. Kwakkel and E. Pruyt (2013). "Adaptive Robust Design under deep uncertainty." Technological Forecasting \& Social Change 80(3).

Howard, R. A., 1988, Decision Analysis: Practice and Promise Management Science 1988 34:679695; doi:10.1287/mnsc.34.6.679

IPCC (2000), Special Report on Emissions Scenarios (SRES). N. Nakicenovic and R. Swart (Eds.), Cambridge University Press, UK. pp 570

IPCC (2007). The IPCC 4th Assessment Report, Technical report, Intergovernmental Panel on Climate Change (IPCC)

Kriegler, E. et al. (2010) Socio-economic Scenario Development for Climate Change Analysis, CIRED Working Paper

Lempert, R. J., B. P. Bryant and S. C. Bankes (2008). Comparing Algorithms for Scenario Discovery. Santa Monica, CA, RAND.

Lempert, R. J., D. G. Groves, S. W. Popper and S. C. Bankes (2006). "A General, Analytic Method for Generating Robust Strategies and Narrative Scenarios." Management Science 52(4): 514-528.

Lempert, R., and N. Kalra (2011), Managing Climate Risks in Developing Countries with Robust Decision MakingRep., World Resources Report, Washington DC.

Lempert, R. J., S. W. Popper, and S. C. Bankes (2003), Shaping the Next One Hundred Years : New Methods for Quantitative, Long-term Policy Analysis, xxi, 187 p. pp., RAND Corporation, Santa Monica, CA. 
Lempert, R. J. (2012), Scenarios that Illuminate Vulnerabilities and Robust Responses, Climatic Change

McJeon, H. C., L. Clarke, P. Kyle, M. Wise, A. Hackbarth, B. Bryant and R. J. Lempert (2011). "Technology Interactions Among Low-Carbon Energy Technologies: What Can We Learn From a Large Number of Scenarios?" Energy Economics 33: 619-631.

Moss, R. H. et al. (2010), The next generation of scenarios for climate change research and assessment. Nature 463, 747-756

O’Neill, B.C., Carter, T., Ebi, K.L., Edmonds, J., Hallegatte, S., Kemp-Benedict, E., Kriegler, E., Mearns, L., Moss, R., Riahi, K., van Ruijven, B., van Vuuren, D. (2012). Meeting Report of the Workshop on The Nature and Use of New Socioeconomic Pathways for Climate Change Research, Boulder, CO, November 2-4, 2011. Available at: http://www.isp.ucar.edu/socio-economic-pathways.

Parson, E. A., V. Burkett, K. Fischer-Vanden, D. Keith, L. Mearns, H. Pitcher, C. Rosenweig, and M. Webster (2006), Global-Change Scenarios: Their Development and Use, Synthesis and Assessment Product 2.1b, public review draft Rep., US Climate Change Science Program.

J. Peace and J. Weyant. Insights not numbers : the appropriate use of economic models, Pew Center on Global Climate Change White Paper, 2008.

Petschel-Held, G., H-J Schellnhuber, T Bruckner, F. L. Tóth, K. Hasselmann (1999). The Tolerable Windows Approach: Theoretical and Methodological Foundations, Climatic Change, 41, Issue 3-4, pp $303-331$

Rozenberg J., S. Hallegatte, A. Vogt-Schilb, O. Sassi, C. Guivarch, H. Waisman and J.-C. Hourcade, (2010), Climate policies as a hedge against the uncertainty on future oil supply. Climatic Change 101(3-4): 663-668

Schweizer, V. J., B. C. O'Neill, Systematic construction of global socioeconomic pathways using internally consistent element combinations, this issue

Toman, M. A., J. Griffin, and R. J. Lempert (2008), Impacts on U.S. energy expenditures and greenhouse-gas emissions of increasing renewable-energy use : technical reportRep. 9780833044976 (pbk. alk. paper), xvii, 54 p. pp, RAND Corp., Santa Monica, CA.

vanVuuren, D. P. et al. (2010), Developing New Scenarios as a Thread for Future Climate Research IPCC Working Paper.

Waisman, H.D., C. Guivarch, F. Grazi, J.-C. Hourcade (2012), The Imaclim-R Model: Infrastructures, Technical Inertia and the Costs of Low Carbon Futures under Imperfect Foresight. Climatic Change (forthcoming) DOI 10.1007/s10584-011-0387-z

Birkmann, J., et al. (2013), Scenarios for Vulnerability, Climate Change, in press.

Garb, Y., S. Pulver, and S. D. VanDeveer (2008), Scenarios in society, society in scenarios: toward a social scientific analysis of storyline-driven environmental modeling, Environ. Res. Lett., 3, 1-8.

Gerst, M. D., P. Wang, and M. E. Borsuk. (2013), Discovering plausible energy and economic futures under global change using multidimensional scenario discovery. , Environmental Modelling \& Software. , 44, 76-86. 
Groves, D. G., and R. J. Lempert (2007), A New Analytic Method for Finding Policy-Relevant Scenarios, Global Environmental Change 17, 73-85.

Haasnoot, M., J. H. Kwakkel, W. E. Walker, and J. ter Maat (2013), Dynamic adaptive policy pathways: A method for crafting robust decisions for a deeply uncertain world, Global Environmental Change, 23(2), 485-498.

Hamarat, C., J. H. Kwakkel, and E. Pruyt (2013), Adaptive Robust Design under deep uncertainty, Technological Forecasting \& Social Change, 80(3).

Kasprzyk, J. R., S. Nataraj, P. M. Reed, and R. J. Lempert (2013), Many-Objective Robust Decision Making for Complex Environmental Systems Undergoing Change, Environmental Modeling and Software, 42, 55-71.

Lempert, R. J., B. P. Bryant, and S. C. Bankes (2008), Comparing Algorithms for Scenario DiscoveryRep., RAND, Santa Monica, CA.

Lempert, R. J., D. G. Groves, S. W. Popper, and S. C. Bankes (2006), A General, Analytic Method for Generating Robust Strategies and Narrative Scenarios, Management Science, 52(4), 514-528.

McJeon, H. C., L. Clarke, P. Kyle, M. Wise, A. Hackbarth, B. Bryant, and R. J. Lempert (2011), Technology Interactions Among Low-Carbon Energy Technologies: What Can We Learn From a Large Number of Scenarios?, Energy Economics, 33, 619-631. 